\title{
Osteodiscitis due to Mycobacterium tuberculosis, in a patient with miliary tuberculosis, left nephrectomy and post-operative wounds infected with multi-drug resistant bacteria
}

\author{
Violeta Melinte1,2, Teodor Vasile ${ }^{3}$, Alexandru-Paul Burcin², Andreea Maria Pirvan², \\ Manuela Nica ${ }^{1,2}$, Simin Florescu ${ }^{1,2}$, Corneliu Popescu ${ }^{1,2}$, Cristiana Oprea ${ }^{1,2}$ \\ 1“Dr. Victor Babes" Clinical Hospital for Infectious and Tropical Diseases, Bucharest, Romania \\ 2"Carol Davila" University of Medicine and Pharmacy, Bucharest, Romania \\ ${ }^{3}$ Hôpital Bicêtre, Paris, France
}

\begin{abstract}
Background. Severe immunosuppression caused by various types of surgical procedures, such as ureteral catheterization, can increase the risk of tuberculosis (TB) reactivation and can be involved in the appereance of disseminated TB (1). Pseudomonas aeruginosa is the third most common organism, after Escherichia coli and Proteus mirabilis, isolated from patients with urinary tract infections in the hospital setting. Despite the progress made in the research field of antibiotics, mortality and morbidity associated with this etiology are still increased (2).

Miliary TB is a form of disseminated TB that can be fatal in the absence of an early diagnosis and treatment. This form of disease mainly affects young immunosuppressed patients, and has a higher prevalence in women $(1,3)$.

Materials and methods. We present the case of a 25 year-old female, who was admitted at Victor Babes Clinical Hospital for Infectious and Tropical Diseases for a postdiscectomy tuberculous spondylodiscitis (T11T12). The hospital admission was for two reasons: to treat the post surgery wounds super infected with methicilin-resistant Staphylococcus aureus and Pseudomonas aeruginosa and secondly, to obtain a bacteriological confirmation for the tuberculous spondylodiscitis (at T11-T12) in order to start an anti-tuberculous drug regimen.

Results. After the diagnosis and treatment initiation with meropenem, colistin, vancomycin and anti-tuberculous drugs the outcome of the patient was favorable. The clinical, bacteriological and radiological parameters normalized after one month of therapy.

Conclusions. When a infection is bacteriologically confirmed, early antibiotic susceptibility testing and treatment are essential for the prevention of serious local and systemic complications associated with surgical procedures.
\end{abstract}

Keywords: tuberculous osteodiscitis, miliary tuberculosis, nephrectomy, post-operative wounds, superinfected, multi-drug resistant, Pseudomonas aeruginosa, methicilin-resistant Staphylococcus aureus (MRSA)

\section{BACKGROUND}

Pseudomonas aeruginosa is frequent the etiologicalagent of catheter associated urinary tract infections and is often resistant to antibiotic treatment due to its ability to form biofilms on the surface of urinary catheters. Initially, the germ forms microcolonies which later coalesce together. Alginate, which is an anionic polysaccharide that can be found in the bacterial wall, is one of the most important virulence factors, and a component of the biofilm. Biofilms are resistant to antimicrobial 
agents as well as to host defense mechanisms, so they are difficult to eradicate. Thus they contribute to persistent and recurrent urinary tract infections (4).

Pseudomonas aeruginosa is often isolated from postsurgical wounds, being the third most frequent after Escherichia coli and Staphylococcus aureus. The incidence of Pseudomonas aeruginosa wound infection is correlated with the age and the sex of the patient, but also with the duration of the hospitalisation.

A study performed by Masaadeh H.A. and Jaran A. concluded that the occurrence of Pseudomonas aeruginosa in postsurgical wound infections was higher in the group of young patients. It showed also that there were no gender differences regarding the susceptibility to infection (5). P. aeruginosa has a multiple resistance profile (6).

Diagnosis of extrapulmonary tuberculosis can be a challenging due to paucibacillary samples. In addition to Mycobacterium tuberculosis cultures, histopathological tests and polymerase chain reaction can be done in order to increase the diagnostic sensitivity (7).

Tuberculous spondylodiscitis affects around $50 \%$ of all patients with musculoskeletal tuberculosis (8). Studies showed that extrapulmonary musculoskeletal TB occurresusually simultaneously with the pulmonary involvement (3). Infection of the spine can develop by haematogenic route or by a lymphogenic pathwaythrough para-aortic lymph nodes. More than one vertebra is usually involved. The bone destruction starts usually at the anterior part of the vertebra, close to the vertebral plates (3). Tuberculous lesions involve the intervertebral disc and the adjacent superior and inferior vertebral bodies but the posterior column is usually spared. The tissue destruction leads to kyphotic deformity (8). Early diagnosis and optimal therapy can reduce the incidence of bone sequalae, such as ankylosis and other deformities, which are frequent if the infection is left untreated. Both early diagnosis and treatment will decrease the number of surgical interventions needed for this particular form of disease (9). A MDR (multi-drug resistance) or XDR (extensive drug resistance) profile can be present among Mycobacterium tuberculosis strains that are frequent in endemic areas such as Romania. This is another issue which can lead to significant difficulties in choosing anti-tuberculous therapy.

\section{CASE REPORT}

A 25 years old female was admitted at Victor Babes Clinical Hospital for Infectious and Tropical Diseases with tuberculous T11-T12 spondylodiscitis after discectomy. There were two reasons for admission, primarily the treatment of surgical wounds infected with Methicilin-Resistant-Staphylococcus aureus and Pseudomonas aeruginosa (only sensitive to Colistin according to drug susceptibility testing) and secondly to obtain a bacteriological confirmation for the T11-T12 tuberculous spondylodiscitis (initially based on histology and Gram stain) and to start an anti-tuberculous drug regimen according to the results.

From her past medical history it's important to mention that two years before the current admission, she had been treated several times in an urology department for recurrent lower urinary tract infections. The onset of the urinary symptomatology was in the 20th week of pregnancy. She was then diagnosed with bilateral hydronephrosis and because of a complicated urinary tract infection, a „double J" catheter was inserted on the right side and a nephrostomy tube on the left side. Several months later, the „double J" catheter was removed but the nephrostomy tube was kept as there was a persistent wound drainage from the left kidney. At that moment, the first urine culture was positive with a Pseudomonas aeruginosa strain susceptible to Colistin, Piperacillin-Tazobactam, TicarcollinClavulanat and resistant to carbapenem, fluoroquinolones and Nitrofurantoin. Due to continuous drainage from the left nephrostomy tube, a renal scintigrafy was performed which revealed a nonfunctional renographic curve of the left kidney, and consequently a left nephrectomy was performed. A chest x-ray performed before the nephrectomy didn't show any changes (Fig. 1).

After the surgical procedure, the patient continued to complain of recurrent wound drainage from the nephrectomy site. Multiple wound swabs were taken and a strain of Pseudomonas aeruginosa sensitive only to Colistin and resistant to antipseudomonal Penicillins was isolated.

Abdominal MRI revealed an abscess located in the left renal fosa. Incision and drainage of the abscess was performed but, with no adequate antibiotic coverage for Pseudomonas aeruginosa, there 


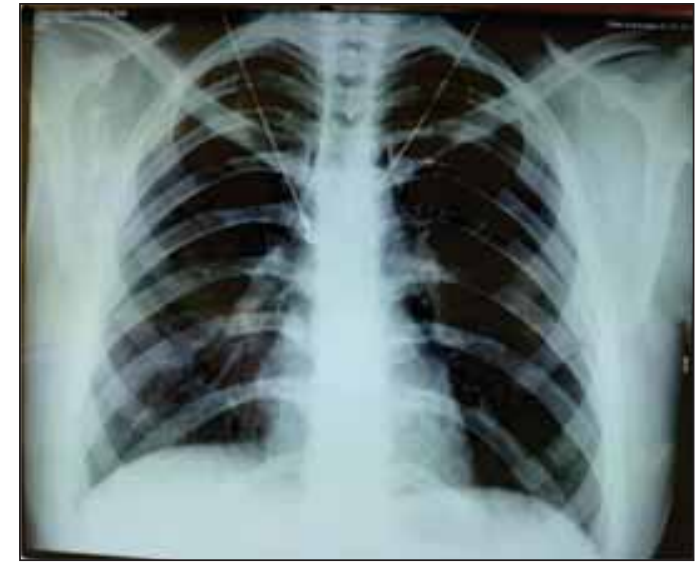

FIGURE 1 a. Chest x-ray before nephrectomy: Normal aspect

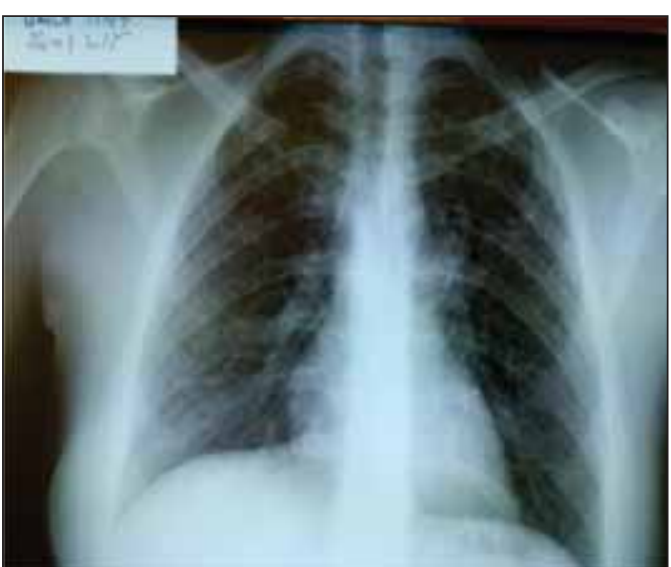

FIGURE 1 b. Chest x-ray at admission: Micronodular opacities in both lung fields

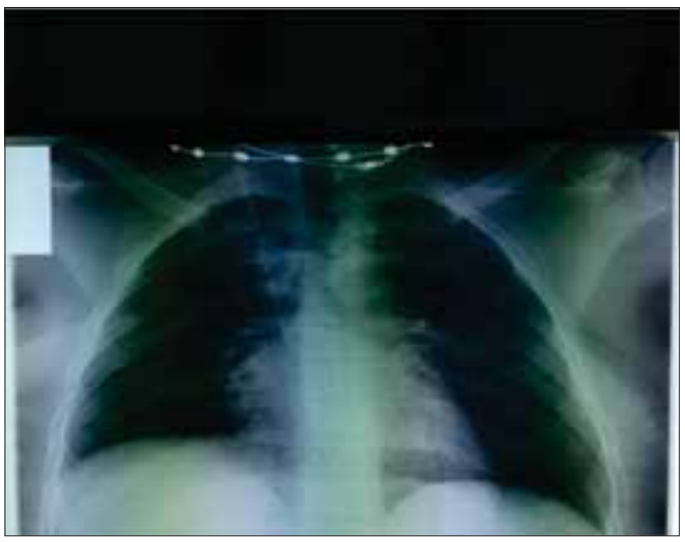

FIGURE 1 c. Chest $x$-ray 3 weeks after treatment initiation: Normal aspect

was recurrent wound drainage from the surgical site. Treatment with Colistin only was started according to the susceptibility test in the urology department.

A month before the admission in our department, the patient complained of recurrent lumbar pain. A spine MRI was performed and showed a lesion suggestive for T11-T12 osteodiscitis (Fig. 2). She was admitted in a neurosurgery department where osteodiscectomy was performed. The histopathological exam revealed a possible tuberculous osteodiscitis, with a granulomatous aspect. ZiehlNeelsen stain yielded the presence of AFB (acidfast bacilli) and was later confirmed by PCR -AND MTB (Fig. 3).

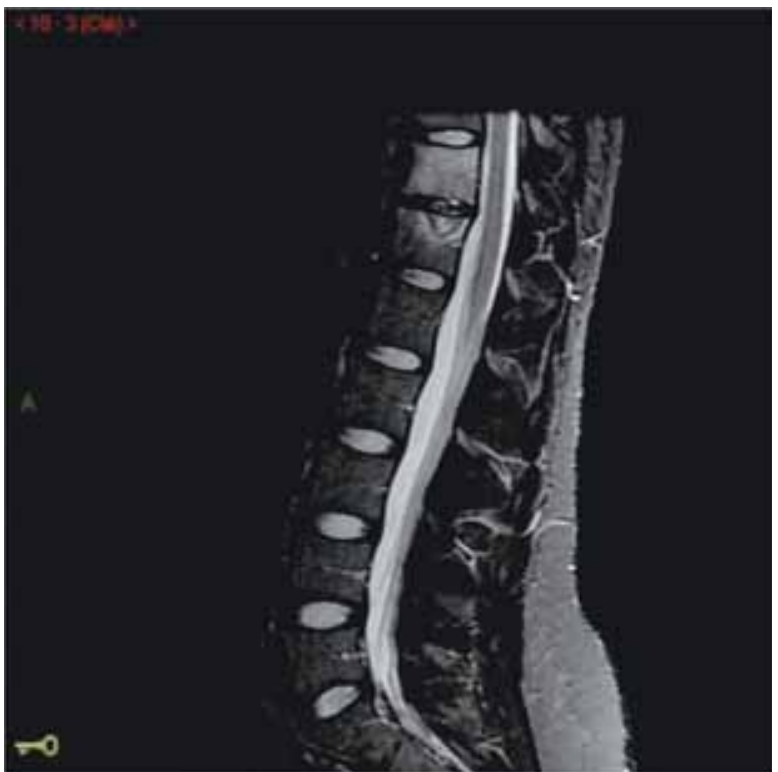

FIGURE 2. MRI (Sagital STIR) - Low signal at T11-T12 level, suggesting an inflammatory process at the level of T11 and T12 vertebral bodies and their intervening disc, with fluid accumulation and adjacent bone marrow edema

At admission, ten days after the procedure, the patient was with moderate altered general status, immobilized in a cast and had fever and pain at the surgical site. At the physical exam a wound with green pus was evidenced at the osteodiscectomy site (Fig. 4a), and an old, incomplete healed scar with pus at the nephrectomy site (Fig. 4b).

Chest x-ray showed multiple nodular opacities in both lungs arguing for miliary tuberculosis (Fig. 1b).

Swab samples from both wounds were taken and cultured and anti-tuberculous treatment was started. The culture from the post nephrectomy lesion was positive with Pseudomonas aeruginosa and Staphylococcus aureus. The swabs from the post osteodiscectomy lesion was also positive with Pseudomonas aeruginosa MDR, sensitive to Colistin only, possibly through contigous spread from the nephrostomy wound.

As both osteodiscitis and urinary tract infections frequently have a common etiology, explained by the local vascular anatomy, a urine PCR test for $M$. tuberculosis was performed in order to rule-out renal tuberculosis and the test was negative. 


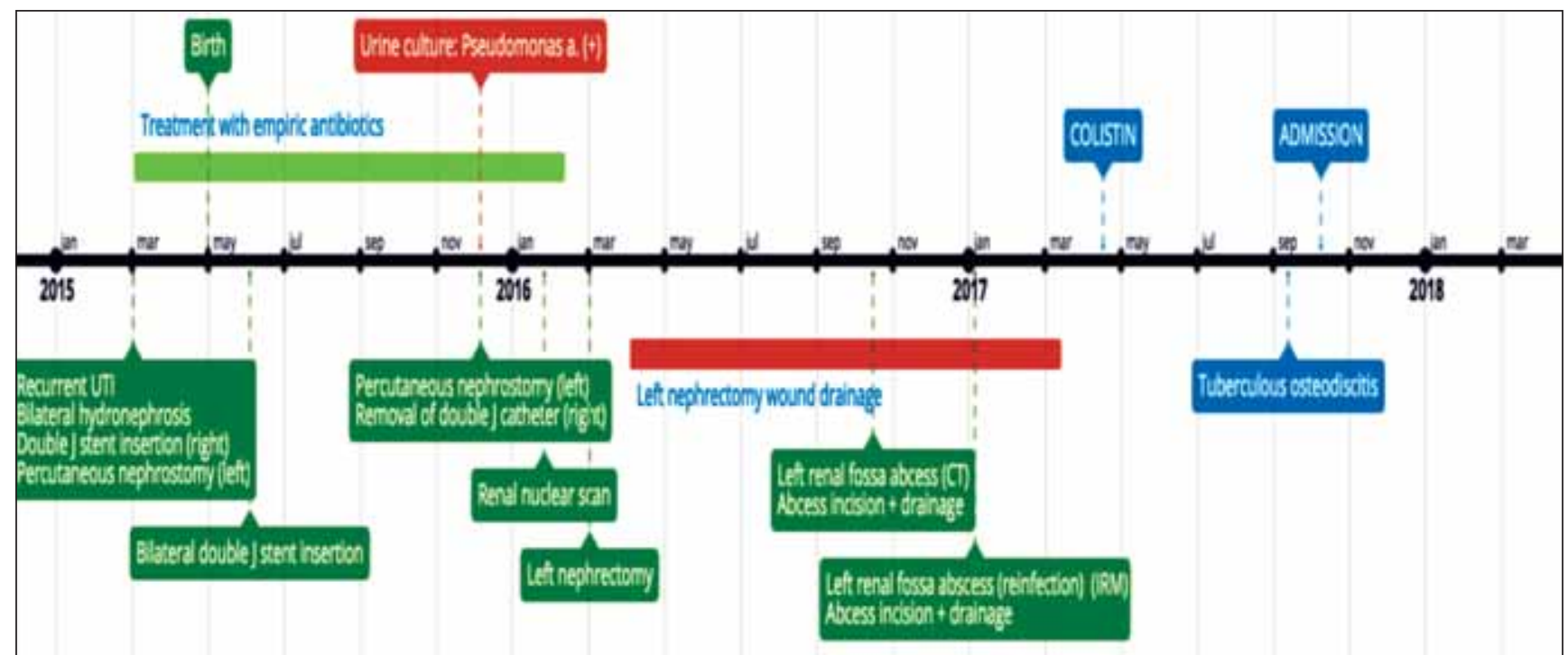

FIGURE 3. Patient history

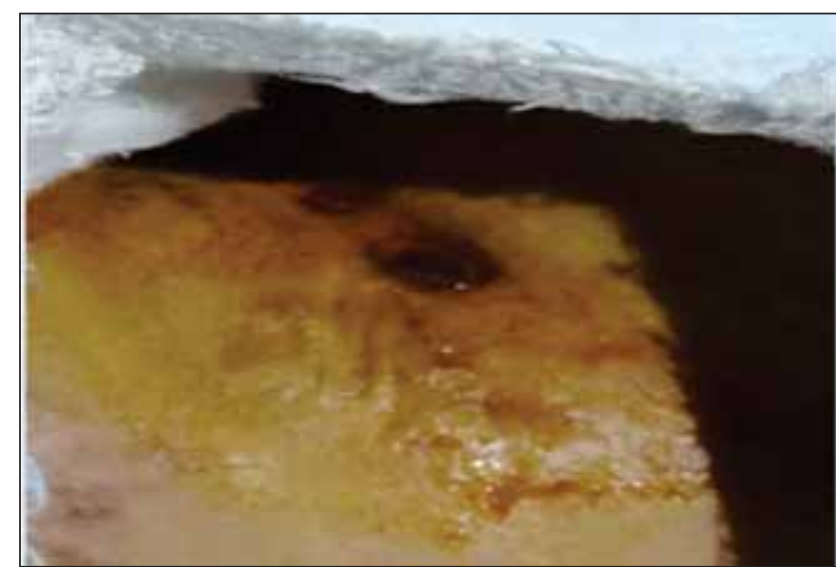

FIGURE 4 a. Infected postnephrectomy wound at admission

Clinical and bacteriological data led to the next diagnsis: tuberculous osteodiscitis at T11-T12 level; miliary tuberculosis; post-operative wounds iin-

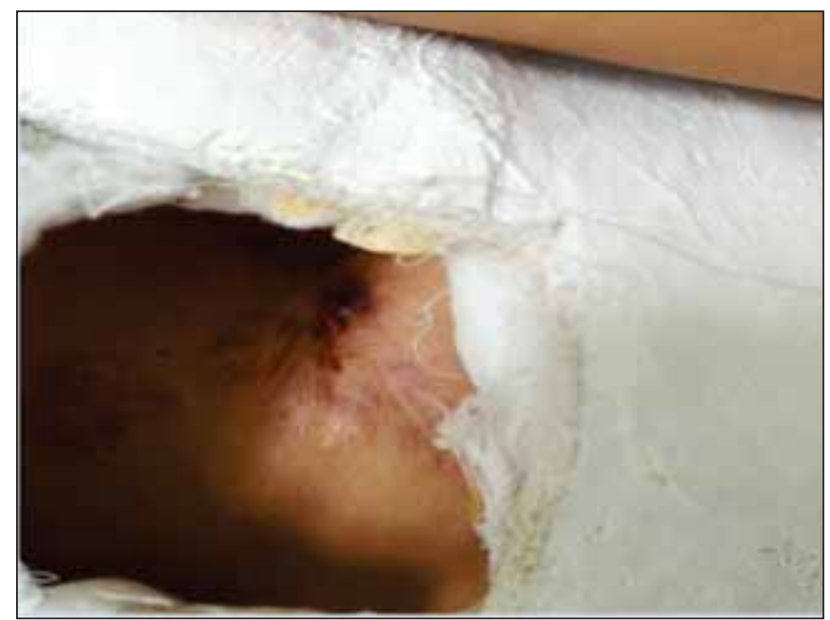

FIGURE 5 a. Healed postnephrectomy wound after treatment initiation

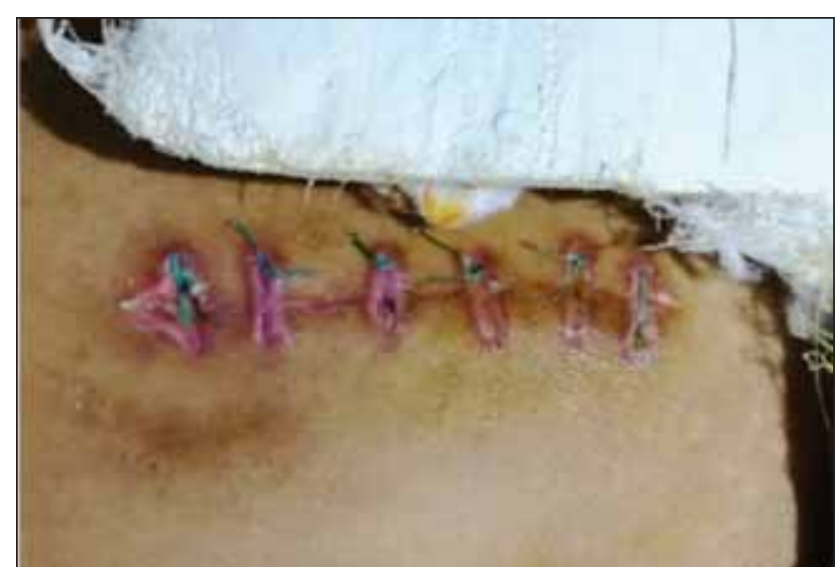

FIGURE 4 b. Superinfected postdiscectomy wound at admission

fected with P. aeruginosa MDR and MRSA; right solitary kidney post left nephrectomy.

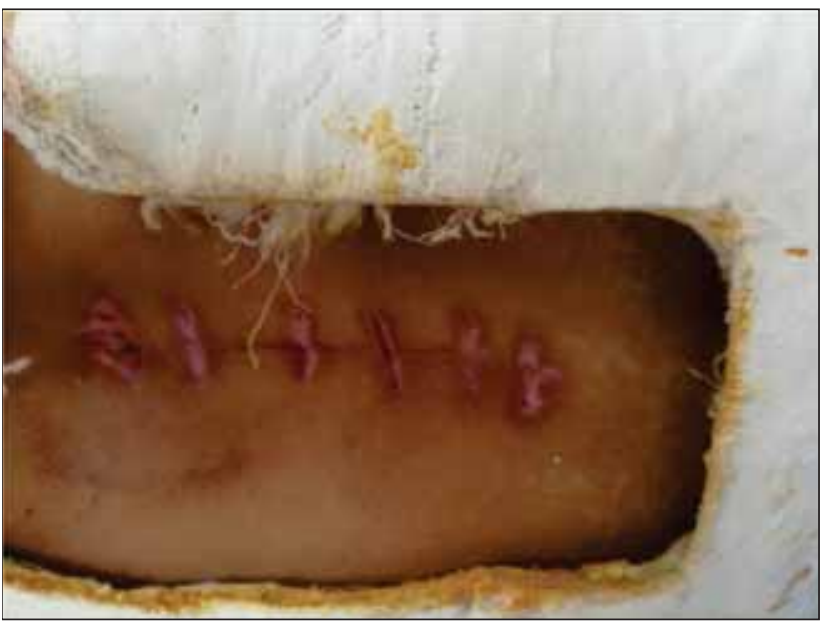

FIGURA 5 b. Healed postdiscectomy wound after treatment initiation 
The patient was started on anti-tuberculous treatment with isoniazid, rifampicin, ethambutol, pyrazinamide and combined antibiotherapy with Colistin, Meropenem and Vancomycin. During the hospitalisation the liver enzymes slightly increased, probably as a secondary effect of the anti-tuberculous drug regimen and the patient reported also a lower exremity pain due to prolonged bed rest. The patient's clinical status improved progressively. The clinical, bacteriological, and imagistic parameters improved under proper antibiotic and anti-tuberculous drug regimens, as well as analgesics, hydration, nutrition, kinetotherapy and psychological support. Both the osteodiscectomy and the nephrostomy wounds showed signs of a normal healing process and no secretions were present after ten days of therapy (Fig. 5 a,b).

The inflammatory response, evaluated by ESR and CRP measurements normalized by the end of the treatment. Vancomycin serum concentrations were also measured for maintaining a buffer interval between maximum treatment efficiency and the lowest possible risk of adverse reactions (Table 1).

The chest $\mathrm{x}$-ray had a normal aspect after aproximately one month of treatment (Fig. $1 \mathrm{c}$ ).

Wound swabs samples were taken after 3 weeks of treatment and showed negative results. The patient was discharged after one month with improved clinical, paraclinical, imagistic and bacteriological parameters.

\section{DISCUSSIONS}

Pseudomonas aeruginosa is a frequent etiologic agent in healthcare-associated urinary tract infections. It is the third most common bacteria in hospital-acquired catheter-associated urinary tract infections after Escherichia coli and the enterococci.
The number of CFU/ml in urine cultures is positively correlated with the duration of the urinary catheterization (4). Environmental factors that lead to urinary infections with Pseudomonas aerugino$s a$ are urinary osmolarity, $\mathrm{pH}$ and Tamm-Horsfall protein, as well as concentration variation of electrolytes such as iron (2). In this case, recurrent urinary tract infections secondary to a gestational hydronephrosis followed by a long period of indwelling catheters usage, led to the loss of left kidney function, followed by nephrectomy.

Repeated drug susceptibility testing showed that there was a progressive pattern of acquired resistance to antibiotics, hence the inability to treat the nephrostomy wound with empiric therapy. At the time of treatment initiation, cultures from both wounds showed a multiresistant strain of Pseudomonas aeruginosa. The meronem-colistin combination was used to obtain a synergistic effect. Through a lipid saponification mechanism, colistin can facilitate meronem penetration through the bacterial wall. According to a cohort study which compared survival rates in a group treated solely with colistin and another group treated with a combinatin of colistin and meronem, the colistin-meronem group of patients had better survival rates (10). A higher treatment efficiency was obtained by vancomycin concentration monitoring. The serum target concentration was $15-20 \mathrm{mg} / \mathrm{dl}$. Two weeks after the admission, there was a complete treatment response according to cultures and susceptibility testing from both wounds.

Osteodiscitis, secondary to Pseudomonas aeruginosa infection was a reasonable hypothesis, considering the persistent urinary cultures with Pseudomonas aeruginosa and the anatomical location of the recurrent wound drainage site. But the

TABLE 1. Dynamic changes of laboratory parameters

\begin{tabular}{|l|c|c|c|c|c|c|c|}
\hline Lab screen & Day 1 & Day 8 & Day 12 & Day 15 & Day 21 & Day 27 & Day 33 \\
\hline Leukocytes $(/ \mu \mathrm{l})$ & 9,500 & 7,700 & & 3,500 & 4,300 & 3,785 & 4,000 \\
\hline Platelets $(/ \mu \mathrm{l})$ & 508,200 & 494,000 & & 361,000 & 281,000 & 253,600 & 233,000 \\
\hline Hemoglobin $(\mathrm{g} / \mathrm{dl})$ & 10.93 & 11 & 10.5 & & & 10 & 11.36 \\
\hline ALT $(\mathrm{U} / \mathrm{l})$ & 34 & & & 171 & 133 & 49 & 28 \\
\hline Creatinine (mg/dl) & 1.2 & 1 & 1 & 1.1 & 0.9 & 0.9 & 0.9 \\
\hline ESR $(\mathrm{mm} / \mathrm{h})$ & 107 & 111 & & 66 & 48 & & 32 \\
\hline CRP $(\mathrm{mg} / \mathrm{dl})$ & 7.8 & 4.16 & & 1.36 & & 0.88 & 1.14 \\
\hline Vancomycin $(\mu \mathrm{g} / \mathrm{ml})$ & & 14.8 & 15.2 & 14.7 & & 21.4 & \\
\hline
\end{tabular}


preoperative chest x-ray showed multiple micronodular opacities in both lung fields. Moreover, the surgical histopathological sample revealed a typical granulomatous pattern, suggestive for tuberculous osteodiscitis which was later confirmed by the presence of AFB in Ziehl-Neelsen stain and positive AND-MTB at PCR testing.

Miliary TB is a disseminated form of tuberculosis that can be fatal in the absence of an early diagnosis and an adequate treatment. Often, tuberculous osteodiscitis is associated with pulmonary TB, in our case a miliary tuberculosis. This form is more common among teenagers and young immunosuppressed adults. Moreover, a higher prevalence of milliary TB has been observed among females $(3,1)$. Some of the predisposing factors for this condition are multiple surgical interventions and procedures such as ureteral catheterization (1).

The 25 years old immunosuppressed female patient has developed tuberculous osteodiscitis by haematogenous spreading, with pulmonary infection being the primary source. The urine PCR analysis was negative for Mycobacterium tuberculosis, hence the possible explanation for this pathogenic mechanism.

\section{REFERENCES}

1. Sharma S.K., Mohan A., Sharma A. Miliary tuberculosis: A new look at an old foe. Journal of Clinical Tuberculosis and Other Mycobacterial Diseases 3, 2016: 13-27

2. Mittal R., Aggarwalc S., Sharmab S., Chhibberb S., Harjaib K. Urinary tract infections caused by Pseudomonas aeruginosa: $A$ minireview. Journal of Infection and Public Health, 2009; 2:101-111

3. Albrecht N., Cottagnoud P., Chatterjee B. Miliary tuberculosis with atypical presentation in an immunocompetent young African man. BMJ Case Rep. 2014.

4. Cole S.J., Records A.R., Orr M.W., Linden S.B., Lee V.T. Catheter-associated urinary tract infection by Pseudomonas aeruginosa is mediated by Exopolysaccharide-independent biofilms. Infect Immun. 2014; 82(5): 2048-2058.

5. Masaadeh H.A., Jaran A.S. Incident of Pseudomonas aeruginosa in Post-Operative Wound Infection. American Journal of Infectious Diseases 5 (1): 1-6, 2009.

6. Jombo G.T., Akpan S., Epoke J., Akaa P.D., Odey F. Multidrug resistant Psudomonasaeruginosa infections complicating surgical wounds and the potential challenges in managing post-operative

\section{CONCLUSIONS}

Long term ureteral catheterization can significantly lower the immune status of a patient. The chronic state of immunosuppression combined with a high endemicity for Mycobacterium tuberculosis can lead to both pulmonary and extrapulmonary disease which may cause severe, lifethreatening complications.

The patient's condition has improved significantly after choosing a correct antibiotic and antituberculous regimen, supported by wound cultures and drug susceptibility testing.

In conclusion, early culture and antibiotic sensitivity testing from surgical wounds, or infected wound drainage sites and long-term indwelling catheters, can lead to more rapid responses to appropriate antimicrobial therapy. This may have a positive impact on morbidity and mortality, which could eventually lead to cost savings by performing fewer tests and shortening the duration of hospitalization.

wound infections: University of Calabar Teaching Hospital experience. Asian Pacific Journal of Tropical Medicine (2010)479-482 479

7. Lee J.Y. Diagnosis and Treatment of Extrapulmonary Tuberculosis, TubercRespir Dis 2015;78:47-55.

8. Koptan W., EIMiligui Y., EISharkawi M. Single stage anterior reconstruction using titanium mesh cages in neglected kyphotictuberculousspondylodiscitis of the cervical spine. Eur Spine J (2011) 20:308-313.

9. Quarta L., Corrado A., Melillo N., Trotta A., Scotto G., D'Onofrio F., Santoro N., Cantatore F.P. Combined effect of Neridronate and specific antibiotic therapy in a case of tuberculous spondylodiscitis. Rheumatollnt (2008) 28:495-498.

10. Maria Helena Rigatto, Fabiane J. Vieira, Laura C. Antochevis, Taina F. Behle, Natane T. Lopes, Alexandre P. Zavascki. Polymyxin B in combination with antimicrobials lacking in vitro activity versus Polymyxin B in monotherapy in critically ill patients with Acinetobacterbaumanii or Pseudomonas aeruginosa infections, antimicrobial agents and chemotherapy. Antimicrobial Agents and Chemotherapy 59:6575-6580. 